\title{
Factors Associated with Delirium in COVID-19 Patients and Their Outcome: A Single-Center Cohort Study
}

\author{
Annabella Di Giorgio ${ }^{1}$, Antonio Mirijello ${ }^{2}$ (D) Clara De Gennaro ${ }^{1}$, Andrea Fontana ${ }^{3}\left(\mathbb{D}\right.$, Paolo Emilio Alboini ${ }^{1}$, \\ Lucia Florio ${ }^{1}$, Vincenzo Inchingolo ${ }^{1}$, Michele Zarrelli ${ }^{1}$, Giuseppe Miscio ${ }^{4}$, Pamela Raggi ${ }^{5}$, \\ Carmen Marciano ${ }^{5}{ }^{\circledR}$, Annibale Antonioni ${ }^{6}{ }^{\circ}$, Salvatore De Cosmo ${ }^{2}$, Filippo Aucella ${ }^{7}$, Antonio Greco ${ }^{8}$, \\ Massimo Carella ${ }^{5} \mathbb{D}$, Massimiliano Copetti ${ }^{3}$ and Maurizio A. Leone ${ }^{1, *,+\mathbb{D}}$ on behalf of the CSS-COVID-19 Group
}

check for

updates

Citation: Di Giorgio, A.; Mirijello, A.; De Gennaro, C.; Fontana, A.; Alboini, P.E.; Florio, L.; Inchingolo, V.; Zarrelli, M.; Miscio, G.; Raggi, P.; et al. Factors Associated with Delirium in COVID-19 Patients and Their Outcome: A Single-Center Cohort Study. Diagnostics 2022, 12, 544 https://doi.org/10.3390/ diagnostics12020544

Academic Editor: Giulia Bivona

Received: 25 January 2022

Accepted: 16 February 2022

Published: 20 February 2022

Publisher's Note: MDPI stays neutral with regard to jurisdictional claims in published maps and institutional affiliations.

Copyright: (C) 2022 by the authors. Licensee MDPI, Basel, Switzerland. This article is an open access article distributed under the terms and conditions of the Creative Commons Attribution (CC BY) license (https:// creativecommons.org/licenses/by/ $4.0 /)$.
1 Neurology Unit, Fondazione IRCCS Casa Sollievo della Sofferenza, 71013 San Giovanni Rotondo, Italy; digiorgioannabella@gmail.com (A.D.G.); clara.degennaro@operapadrepio.it (C.D.G.); paoloemilio.alboini84@gmail.com (P.E.A.); luciaflorio81@gmail.com (L.F.); v.inchingolo@gmail.com (V.I.); m.zarrelli@operapadrepio.it (M.Z.)

2 Internal Medicine Unit, Fondazione IRCCS Casa Sollievo della Sofferenza, 71013 San Giovanni Rotondo, Italy; a.mirijello@operapadrepio.it (A.M.); s.decosmo@operapadrepio.it (S.D.C.)

3 Biostatistics Unit, Fondazione IRCCS Casa Sollievo della Sofferenza, 71013 San Giovanni Rotondo, Italy; a.fontana@operapadrepio.it (A.F.); m.copetti@operapadrepio.it (M.C.)

4 Laboratory Medicine Unit, Fondazione IRCCS Casa Sollievo della Sofferenza, 71013 San Giovanni Rotondo, Italy; g.miscio@operapadrepio.it

5 Scientific Research Department, Fondazione IRCCS Casa Sollievo della Sofferenza, 71013 San Giovanni Rotondo, Italy; p.raggi@operapadrepio.it (P.R.); carmen-marciano@libero.it (C.M.); m.carella@operapadrepio.it (M.C.)

6 Unit of Clinical Neurology, Department of Neuroscience and Rehabilitation, AOU Sant'Anna, 44124 Ferrara, Italy; annibaleantonioni658@gmail.com

7 Nephrology Unit, Fondazione IRCCS Casa Sollievo della Sofferenza, 71013 San Giovanni Rotondo, Italy; f.aucella@operapadrepio.it

8 Geriatric Unit, Fondazione IRCCS Casa Sollievo della Sofferenza, 71013 San Giovanni Rotondo, Italy; a.greco@operapadrepio.it

* Correspondence: m.leone@operapadrepio.it; Tel.: +39-0882-410964

+ Other members of the CSS-COVID-19 Group are indicated in Appendix A.

Abstract: Background: A significant proportion of patients with coronavirus disease 2019 (COVID-19) suffer from delirium during hospitalization. This single-center observational study investigates the occurrence of delirium, the associated risk factors and its impact on in-hospital mortality in an Italian cohort of COVID 19 inpatients. Methods: Data were collected in the COVID units of a general medical hospital in the South of Italy. Socio-demographic, clinical and pharmacological features were collected. Diagnosis of delirium was based on a two-step approach according to 4AT criteria and DSM5 criteria. Outcomes were: dates of hospital discharge, Intensive Care Unit (ICU) admission, or death, whichever came first. Univariable and multivariable proportional hazards Cox regression models were estimated, and risks were reported as hazard ratios (HR) along with their $95 \%$ confidence intervals ( $95 \% \mathrm{CI}$ ). Results: A total of $47 / 214$ patients (22\%) were diagnosed with delirium (21 hypoactive, 15 hyperactive, and 11 mixed). In the multivariable model, four independent variables were independently associated with the presence of delirium: dementia, followed by age at admission, C-reactive protein (CRP), and Glasgow Coma Scale. In turn, delirium was the strongest independent predictor of death/admission to ICU (composite outcome), followed by Charlson Index (not including dementia), CRP, and neutrophil-to-lymphocyte ratio. The probability of reaching the composite outcome was higher for patients with the hypoactive subtype than for those with the hyperactive subtype. Conclusions: Delirium was the strongest predictor of poor outcome in COVID-19 patients, especially in the hypoactive subtype. Several clinical features and inflammatory markers were associated with the increased risk of its occurrence. The early recognition of these factors may help clinicians to select patients who would benefit from both non-pharmacological and pharmacological interventions in order to prevent delirium, and in turn, reduce the risk of admission to ICU or death. 
Keywords: delirium; COVID-19; mortality; C-reactive protein; neutrophils-to lymphocyte ratio

\section{Introduction}

Delirium is a neurocognitive disorder characterized by disturbance in attention and awareness that develops over a short period of time (hours, days), fluctuates, and represents a change from the baseline behavioral state as a consequence of an underlying medical condition [1]. Its occurrence is the highest among hospitalized older individuals. Delirium has been associated with high mortality, increased morbidity, functional decline, extended length of hospital stay and increased requirement for institutional care [2]. Features of delirium predicting worse outcomes include older age, frailty, hypoactive subtype, and delirium severity and duration2. Previous studies in patients with coronavirus disease 2019 (COVID-19) demonstrated that 20-30\% of patients present with or develop delirium during their hospitalization [3], especially in the elderly [4,5], with rates of $55-70 \%$ in cases of severe illness [6]. However, although delirium has emerged as a major complication in the clinical management of COVID-19 patients, there has been a lack of attention paid to its identification, to the ascertainment of its risk factors and to the impact of its outcomes in COVID-19 patients [7]. Reasons for this include the various definitions of delirium, the absence of a systematic assessment for delirium in current COVID-19 management guidelines, the retrospective nature of the studies, the different clinical classification of delirium, the lack of recognition of delirium as an atypical presentation of the disease, and the failure to account for delirium's impact on mortality [8]. Two were the objectives of the present study: first, to evaluate the occurrence of delirium in a large cohort of adults hospitalized for COVID-19 and its impact on in-hospital mortality; second, to identify risk factors associated with delirium; in this regard, our attention was focused on inflammatory biomarkers. These biomarkers are particularly important, as immunopathology has been suggested as a primary driver of morbidity and mortality with COVID-19 [9].

\section{Materials and Methods}

\subsection{Design and Sample}

This was a single-center observational study conducted at the "IRCCS Casa Sollievo della Sofferenza" hospital, a 900-bed general hospital with a catchment area of about 300,000 inhabitants, in Southern Italy. From 3 March to 31 May 2020, all consecutive patients aged $\geq 18$ years suspected of COVID-19 infection were admitted to our COVID units (internal medicine and geriatric units). All patients had epidemiologic, clinical, laboratory and radiological findings suspected for COVID-19 [10]. Real-time reversetranscriptase polymerase chain reaction from nasopharyngeal swab was performed in all patients and repeated, in the case of a negative result, as appropriate. The study was performed in accordance with the Declaration of Helsinski of 1964 and all its subsequent amendments and revisions, and it was approved by the local Institutional Ethics Committee (COVID-19-SGR-46/2020). Written informed consent was obtained from all participants or from their next of kin for those incapable of providing the informed consent. Inclusion criteria were: informed consent, age $\geq 18$-year-old, and admission to a COVID Unit with suspected or proven SARS-CoV-2 infection. The exclusion criteria were: (1) a previous diagnosis of major psychiatric disorders (schizophrenia spectrum disorders), and (2) the impossibility to evaluate the delirium due to precipitating medical conditions, death, or missing charts.

\subsection{Patient and Public Involvement}

Patients and the public were not involved in any way in the design and conduct of the study, in the choice of outcome measures or in the recruitment. 


\subsection{Data Collection}

Data were retrieved from clinical records and entered in an electronic clinical record form. Demographic data (age at admission, gender, education, marital status, employment), type and date of onset of symptoms, date of hospital admission, number and type of comorbidity and chronic medications, baseline clinical data at admission (blood pressure, BMI, Glasgow Coma scale-GCS, symptoms), and smoking status were collected. The Charlson Comorbidity Index [11] was calculated to determine the burden of comorbidity; however, in the time-to-event analyses the dementia score was subtracted from the total score because dementia had been already included as a covariate. ECG (with the measure of QTc), arterial blood gas analysis, chest X-ray, and chest CT were also performed. Laboratory tests were carried out at admission to the emergency department or within $24 \mathrm{~h}$ and included: blood cell count, activated partial thromboplastin time, International Normalized Ratio (INR), alkaline phosphatase, total bilirubin, aspartate and ala-nine-aminotransferase, r-glutamyl-transpeptidase, total proteins, creatinine, estimated glomerular filtration rate (eGFR), glycemia, erythrocyte sedimentation rate, triglycerides, cholesterol, lactate-dehydrogenase (LDH), creatine-phosphokinase, troponin, D-dimer, procalcitonin, and C-reactive protein (CRP). The neutrophils-to-lymphocytes ratio (NLR) value was calculated as the absolute periphery neutrophil count divided by the absolute periphery lymphocyte count. Clinical data were collected from the patients and implemented with interviews with relatives or care-givers. The diagnosis of delirium (with the date of onset) involved a two-step process: a brief screening based on the evaluation of nursing and doctors' daily reports in accordance with the criteria of the 4AT tool (alertness, orientation, attention, and acute change or fluctuating course) [12], followed in the case of positivity by a bedside psychiatric evaluation (A.DG.) based on DSM- 5 criteria, in order to confirm or not the diagnosis of delirium. In the case of onset before admission, the date of onset was ascertained through interviews with relatives or caregivers. Once diagnosed, delirium was further clinically classified based upon the predominant motor activity profile into three subtypes: hypoactive, hyperactive, and mixed [13]. The hypoactive and the hyperactive subtypes are characterized by decreased and increased motor activity, respectively, while the mixed subtype by features of both subtypes within short time frames. The following outcomes were retrieved from electronic medical records: dates of hospital discharge, Intensive Care Unit (ICU) admission, or death.

\subsection{Statistical Analysis}

Patients' baseline demographical and clinical characteristics are reported as median and range or as mean and standard deviation for continuous variables (according to their distribution), and as frequencies and percentages for categorical variables. The normal distribution assumption was tested using Shapiro-Wilk and Kolmogorov-Smirnov tests, as well as by graphical inspection of the $Q-Q$ plot. The baseline variables were compared between patients who developed delirium and those who did not, using the Mann-Whitney test and the chi-square test for crude comparisons, and analysis of covariance (ANCOVA) or binary logistic regression for age- and sex-adjusted comparisons. Time to onset of delirium was calculated from the first COVID-19-related symptom to the onset of delirium, or discharge, whichever came first. Overall survival was defined as the time between admission and death. For subjects who were still alive, survival time was censored at discharge. Survival curves were estimated by using the Kaplan-Meier method and compared by the log-rank test. For time to event endpoints, univariable and multivariable proportional hazards Cox regression models were estimated, and risks were reported as hazard ratios (HR) along with their 95\% confidence intervals (95\% CI). The proportional hazards assumption was tested using Schoenfeld residuals. A stepwise variables selection was used to build multivariable models. A two-sided $p$ value $<0.05$ was considered for statistical significance. Univariate Cox regression analyses were adjusted for multiple comparisons using the Bonferroni method. All statistical analyses were performed using the computing environment $R$ ( $R$ Development Core Team, version 3.3.2). 


\section{Results}

Of the 254 consecutive patients admitted for suspected COVID-19 infection, 22 had a final diagnosis of non-COVID-19 infection and were excluded. Of the remaining 232, there were 169 swab-confirmed diagnoses, 26 antibody-confirmed diagnoses, and 37 patients showed clinical and radiological features of COVID-19 despite negative swabs [10]. For 17 of them the evaluation of delirium was impossible (death before assessment, or missing clinical charts). Thus, we ultimately had 214 patients available for analysis, and $47(22 \%)$ were diagnosed with delirium. In 20 patients, delirium was already present at admission, mostly for 1-2 days, whereas in the remaining 27 patients it developed during hospital stay ( 1 to 72 days after admission). Twenty-one patients presented the hypoactive type, 15 the hyperactive type, and 11 patients showed the mixed type. Demographics and clinical features at admission are displayed in Table 1. Baseline laboratory findings are shown in Table 2.

Table 1. Frequency of demographics and clinical characteristics at admission in COVID-19 patients with and without delirium.

\begin{tabular}{|c|c|c|c|}
\hline & Total $(\mathrm{N}=214)$ & $\begin{array}{c}\text { Without } \\
\text { Delirium }(N=168)\end{array}$ & $\begin{array}{l}\text { With Delirium } \\
\quad(\mathrm{N}=46)\end{array}$ \\
\hline Age at admission: mean (SD) & $67.88(15.05)$ & $64.32(14.43)$ & $80.89(8.89)$ \\
\hline \multicolumn{4}{|l|}{ Age class $(\mathrm{N}, \%)$} \\
\hline$<50$ & $29(13.6 \%)$ & $29(17.3 \%)$ & $0(0.0 \%)$ \\
\hline $50-59$ & $32(15.0 \%)$ & $31(18.5 \%)$ & $1(2.2 \%)$ \\
\hline $60-69$ & $44(20.6 \%)$ & $40(23.8 \%)$ & $4(8.7 \%)$ \\
\hline $70-79$ & $59(27.6 \%)$ & $43(25.6 \%)$ & $16(34.8 \%)$ \\
\hline$\geq 80$ & $50(23.4 \%)$ & $25(14.9 \%)$ & $25(54.3 \%)$ \\
\hline Male gender $(\mathrm{N}, \%)$ & $118(55.1 \%)$ & $97(57.7 \%)$ & $21(45.7 \%)$ \\
\hline \multicolumn{4}{|l|}{ Education (years) } \\
\hline$\leq 5$ & $88(41.5 \%)$ & $55(33.1 \%)$ & $33(71.7 \%)$ \\
\hline$\overline{6}-8$ & $55(25.9 \%)$ & $48(28.9 \%)$ & $7(15.2 \%)$ \\
\hline$>8$ & $69(32.5 \%)$ & $63(38.0 \%)$ & $6(13.0 \%)$ \\
\hline Smokers (N, \%) & $44(27.2 \%)$ & $40(30.5 \%)$ & $4(12.9 \%)$ \\
\hline BMI: mean (SD) & $26.97(4.62)$ & $26.98(4.59)$ & $26.89(4.84)$ \\
\hline Glasgow Coma Scale mean (SD) & $14.45(2.06)$ & $14.71(1.64)$ & $13.47(3.00)$ \\
\hline Fever at admission $(\mathrm{N}, \%)$ & $154(72.0 \%)$ & $128(76.2 \%)$ & $26(56.5 \%)$ \\
\hline \multicolumn{4}{|l|}{ Comorbidity at admission (N, \%) } \\
\hline Dementia & $33(15.5 \%)$ & $9(5.4 \%)$ & $24(52.2 \%)$ \\
\hline $\begin{array}{c}\text { Chronic obstructive pulmonary } \\
\text { disease }\end{array}$ & $23(10.7 \%)$ & $17(10.1 \%)$ & $6(13.0 \%)$ \\
\hline Diabetes & $40(18.7 \%)$ & $27(16.1 \%)$ & $13(28.3 \%)$ \\
\hline Atrial fibrillation & $29(13.6 \%)$ & $21(12.5 \%)$ & $8(17.4 \%)$ \\
\hline Arterial hypertension & $103(48.1 \%)$ & $77(45.8 \%)$ & $26(56.5 \%)$ \\
\hline Hypercholesterolemia & $17(7.9 \%)$ & $16(9.5 \%)$ & $1(2.2 \%)$ \\
\hline Tumor & $40(18.7 \%)$ & $33(19.6 \%)$ & $7(15.2 \%)$ \\
\hline Thyreopathy & $17(7.9 \%)$ & $14(8.3 \%)$ & $3(6.5 \%)$ \\
\hline Hypertriglyceridemia & $7(3.3 \%)$ & $5(3.0 \%)$ & $2(4.3 \%)$ \\
\hline Others & $140(65.4 \%)$ & $99(58.9 \%)$ & $41(89.1 \%)$ \\
\hline $\begin{array}{l}\text { Charlson Index (without dementia) } \\
\text { mean (SD) }\end{array}$ & $3.58(2.40)$ & $3.15(2.38)$ & $5.15(1.71)$ \\
\hline Use of psychotropic drugs $(\mathrm{N}, \%)$ & $27(12.6 \%)$ & $14(8.3 \%)$ & $13(28.3 \%)$ \\
\hline
\end{tabular}


Table 2. Baseline + laboratory findings in COVID-19 patients with and without delirium.

\begin{tabular}{|c|c|c|c|}
\hline & Total $(N=214) \ddagger$ & $\begin{array}{c}\text { Without } \\
\text { Delirium }(N=168) \ddagger\end{array}$ & $\begin{array}{l}\text { With Delirium } \\
\qquad(\mathrm{N}=46) \ddagger\end{array}$ \\
\hline $\begin{array}{l}\text { Red blood cells (RBC) } \\
\text { millions } / \mathrm{mcl}\end{array}$ & $4.58(3.97,5.09)$ & $4.68(4.13,5.14)$ & $4.09(3.70,4.83)$ \\
\hline Hematocrit \% & $40.25(35.02,43.98)$ & $40.80(36.02,44.08)$ & $36.25(32.95,42.95)$ \\
\hline Hemoglobin g/dL & $13.30(11.40,14.50)$ & $13.40(11.90,14.70)$ & $11.80(10.78,13.72)$ \\
\hline $\begin{array}{l}\text { Mean corpuscular volume } \\
\text { (MCV) fl }\end{array}$ & $87.80(84.68,91.12)$ & $87.30(83.97,91.03)$ & $89.20(86.45,91.82)$ \\
\hline $\begin{array}{l}\text { White blood cells (WBC) } \\
\text { thousand } / \mathrm{mcl}\end{array}$ & $6.39(4.60,9.04)$ & $5.92(4.34,8.10)$ & $8.29(5.54,11.18)$ \\
\hline Neutrophils thousand/mcl & $4.54(3.04,7.68)$ & $4.19(2.89,6.39)$ & $6.61(4.28,9.27)$ \\
\hline Lymphocytes thousand/mcl & $1.02(0.73,1.45)$ & $1.02(0.77,1.46)$ & $1.06(0.68,1.42)$ \\
\hline $\begin{array}{l}\text { Neutrophils-to-lymphocytes } \\
\text { ratio (NLR) }\end{array}$ & $4.18(2.52,7.41)$ & $3.84(2.40,6.52)$ & $6.04(3.19,18.89)$ \\
\hline Platelets thousand/mcl & $212.00(159.25,285.50)$ & $205.00(153.25,258.00)$ & $273.00(197.50,332.25)$ \\
\hline $\begin{array}{l}\text { International normalized ratio } \\
\text { (INR) }\end{array}$ & $1.08(1.04,1.15)$ & $1.07(1.03,1.13)$ & $1.13(1.06,1.19)$ \\
\hline $\begin{array}{l}\text { Partial thromboplastin time } \\
\text { (PTT) sec }\end{array}$ & $24.60(22.90,26.90)$ & $24.60(22.90,26.90)$ & $24.45(22.10,28.00)$ \\
\hline Alkaline phosphatase UI/L & $74.00(60.00,101.50)$ & $71.00(59.25,96.75)$ & $85.00(70.00,121.00)$ \\
\hline Total bilirubin mg/dL & $0.60(0.40,0.80)$ & $0.60(0.40,0.80)$ & $0.60(0.40,0.90)$ \\
\hline $\begin{array}{l}\text { Aspartate amino transferase } \\
\text { (AST) UI/L }\end{array}$ & $33.00(22.00,48.00)$ & $33.00(23.00,48.00)$ & $32.00(18.50,47.50)$ \\
\hline $\begin{array}{l}\text { Alanine amino transferase } \\
\text { (ALT) } U / L\end{array}$ & $30.00(20.00,48.00)$ & $32.50(21.00,51.00)$ & $24.00(16.00,38.00)$ \\
\hline $\begin{array}{l}\text { y-glutamyl-transpeptidase } \\
(\mathrm{y}-\mathrm{GT}) \mathrm{UI} / \mathrm{L}\end{array}$ & $46.00(22.75,103.00)$ & $51.50(23.00,109.50)$ & $35.50(17.25,62.75)$ \\
\hline Total proteins $\mathrm{g} / \mathrm{dL}$ & $7.00(6.40,7.70)$ & $7.15(6.50,7.80)$ & $6.70(6.10,7.20)$ \\
\hline Creatinine mg/dL & $0.90(0.70,1.10)$ & $0.80(0.70,1.10)$ & $1.10(0.70,1.60)$ \\
\hline $\begin{array}{l}\text { Estimated glomerular filtration } \\
\text { rate (eGFR), } \mathrm{mL} / \mathrm{mn}\end{array}$ & $86.00(58.00,108.75)$ & $90.00(66.75,110.00)$ & $61.50(33.50,97.25)$ \\
\hline Glycemia mg/dL & $106.50(85.00,135.00)$ & $104.50(85.00,127.75)$ & $120.50(92.00,163.00)$ \\
\hline $\begin{array}{l}\text { Erythrocyte sedimentation rate } \\
\text { (ESR) } \mathrm{mm}\end{array}$ & $45.00(30.00,67.00)$ & $44.00(29.75,65.50)$ & $51.00(42.00,70.00)$ \\
\hline Triglycerides mg/dL & $110.00(88.50,146.00)$ & $109.00(84.00,145.00)$ & $116.00(106.25,169.25)$ \\
\hline Cholesterol mg/dL & $137.00(111.50,164.00)$ & $137.00(112.00,164.00)$ & $128.50(105.00,155.25)$ \\
\hline $\begin{array}{l}\text { Lactate-dehydrogenase } \\
\text { (LDH) U/L }\end{array}$ & $243.00(194.00,318.50)$ & $250.50(196.25,326.75)$ & $227.00(180.00,287.00)$ \\
\hline $\begin{array}{c}\text { Creatine-phosphokinase CK } \\
\text { U/L }\end{array}$ & $83.00(46.00,167.00)$ & $80.00(50.00,169.00)$ & $88.00(36.00,154.00)$ \\
\hline Troponin pg/mL & $15.10(8.00,41.60)$ & $12.60(7.40,22.90)$ & $38.85(16.12,143.18)$ \\
\hline D-dimer mg/mL & $764.00(395.00,2981.50)$ & $710.00(393.50,2136.25)$ & $2345.00(505.00,6021.00)$ \\
\hline Procalcitonin $\mu \mathrm{g} / \mathrm{L}$ & $0.14(0.08,0.31)$ & $0.12(0.07,0.27)$ & $0.23(0.12,0.42)$ \\
\hline $\begin{array}{l}\text { C-reactive protein (CRP) } \\
\mathrm{mg} / \mathrm{dL}\end{array}$ & $4.93(1.52,11.05)$ & $3.59(1.07,9.04)$ & $10.705 .73,15.75)$ \\
\hline
\end{tabular}

† Laboratory tests were performed at the emergency department, or within $24 \mathrm{~h}$ after admission; $\ddagger$ Values are reported as median $(\mathrm{Q} 1, \mathrm{Q})$.

In the univariable model, after adjustment for multiple comparisons, the statistically significant predictors of delirium development were: age, GCS, Charlson Index without dementia, dementia, pre-admission use of psychotropic drugs, CRP and NLR (Table 3). 
Table 3. Association between baseline clinical and laboratory variables and the diagnosis of delirium.

\begin{tabular}{|c|c|c|c|c|c|}
\hline & & & $\begin{array}{l}\text { Occurrence of } \\
\text { Delirium }\end{array}$ & & \\
\hline Variable & $\mathbf{N}+$ & N Events $\ddagger$ & $\begin{array}{l}\text { Hazard Ratio (95\% } \\
\text { Confidence Interval) }\end{array}$ & $p$-Value & $\begin{array}{c}p \text {-Value } \\
\text { Adjusted * }\end{array}$ \\
\hline Age $^{\wedge}$ & 214 & 46 & $1.11(1.08-1.15)$ & $1.15 \times 10^{-10}$ & $5.51 \times 10^{-9}$ \\
\hline Male gender & 214 & 46 & $0.67(0.38-1.2)$ & 0.182434625 & 1 \\
\hline Current/former smokers & 162 & 31 & $0.42(0.15-1.21)$ & 0.107783665 & 1 \\
\hline $\mathrm{BMI}^{\wedge}$ & 192 & 34 & $1.01(1-1.02)$ & 0.041076706 & 1 \\
\hline Glasgow Coma Scale^ & 205 & 43 & $0.86(0.79-0.93)$ & 0.00028387 & 0.013625766 \\
\hline Fever & 214 & 46 & $0.43(0.24-0.76)$ & 0.004144349 & 0.198928741 \\
\hline $\begin{array}{c}\text { Chronic obstructive pulmonary } \\
\text { disease }\end{array}$ & 214 & 46 & $1.21(0.51-2.87)$ & $6.60 \times 10^{-1}$ & 1.00 \\
\hline Diabetes & 214 & 46 & $1.99(1.04-3.8)$ & 0.036791187 & 1 \\
\hline Atrial fibrillation & 214 & 46 & $1.39(0.65-2.97)$ & 0.402521663 & 1 \\
\hline Arterial hypertension & 214 & 46 & $1.48(0.83-2.66)$ & 0.184784901 & 1 \\
\hline Hypercholesterolemia & 214 & 46 & $0.21(0.03-1.54)$ & 0.125310853 & 1 \\
\hline Neoplasia & 214 & 46 & $0.71(0.32-1.59)$ & 0.401540797 & 1 \\
\hline Thyreopathy & 214 & 46 & $0.91(0.28-2.94)$ & 0.875627181 & 1 \\
\hline Hypertriglyceridemia & 214 & 46 & $1.32(0.32-5.47)$ & 0.705744187 & 1 \\
\hline Other comorbidities & 214 & 46 & $5.32(2.09-13.51)$ & 0.000439886 & 0.02111453 \\
\hline $\begin{array}{c}\text { Charlson Index (without } \\
\text { dementia) }\end{array}$ & 214 & 46 & $1.35(1.2-1.53)$ & $6.39966 \times 10^{-7}$ & $3.07184 \times 10^{-5}$ \\
\hline Use of psychotropic drugs & 214 & 46 & $3.58(1.87-6.83)$ & 0.000112691 & 0.005409186 \\
\hline Dementia & 213 & 46 & $11.21(6.13-20.5)$ & $4.17 \times 10^{-15}$ & $2.00 \times 10^{-13}$ \\
\hline Vit. D therapy & 214 & 46 & $4.28(1.68-10.91)$ & $2.34 \times 10^{-3}$ & 0.11250987 \\
\hline Neutrophil/lymphocyte ratio $\S$ & 210 & 44 & $1.45(1.21-1.73)$ & $4.87904 \times 10^{-5}$ & 0.002341939 \\
\hline Hematocrit $\$$ & 210 & 44 & $0.67(0.43-1.03)$ & 0.067830978 & 1 \\
\hline Hemoglobin $\S$ & 210 & 44 & $0.18(0.05-0.69)$ & 0.012064584 & 0.579100049 \\
\hline $\mathrm{MCV} \S$ & 204 & 40 & $1.36(0.88-2.1)$ & 0.160177209 & 1 \\
\hline $\mathrm{RBC} \S$ & 210 & 44 & $0.28(0.01-9.86)$ & 0.480843162 & 1 \\
\hline WBC $\S$ & 210 & 44 & $1.35(1.02-1.79)$ & 0.03343282 & 1 \\
\hline Neutrophils $\S$ & 210 & 44 & $1.11(0.93-1.31)$ & 0.243821628 & 1 \\
\hline Lymphocytes $\S$ & 210 & 44 & $0.22(0.01-4.87)$ & 0.337755457 & 1 \\
\hline Platelets $\S$ & 210 & 44 & $1.04(1.01-1.07)$ & 0.002220278 & 0.106573321 \\
\hline INR $\S$ & 194 & 38 & $6.74(0.15-310.51)$ & 0.32861737 & 1 \\
\hline PTT $\S$ & 194 & 38 & $1.13(0.61-2.1)$ & 0.703355655 & 1 \\
\hline ALT $\S$ & 205 & 43 & $0.95(0.87-1.04)$ & 0.244626107 & 1 \\
\hline Total bilirubin $\S$ & 202 & 41 & $2.81(0.07-110.22)$ & 0.581749862 & 1 \\
\hline Alkaline phosphatase ${ }^{\S}$ & 163 & 37 & $1.03(0.98-1.08)$ & 0.23127989 & 1 \\
\hline $\mathrm{r}-\mathrm{GT} \S$ & 164 & 36 & $1(0.97-1.03)$ & 0.820668706 & 1 \\
\hline AST $\S$ & 205 & 43 & $0.97(0.9-1.05)$ & 0.44704993 & 1 \\
\hline Total proteins $\S$ & 203 & 41 & $0.03(0-1.05)$ & 0.053086568 & 1 \\
\hline Creatinine $\S$ & 206 & 43 & $1.05(0.4-2.78)$ & 0.923433337 & 1 \\
\hline $\mathrm{e}-G F R \S$ & 202 & 42 & $0.9(0.83-0.97)$ & 0.004767905 & 0.228859456 \\
\hline Glycemia $\S$ & 204 & 42 & $1.06(1.03-1.11)$ & 0.000990944 & 0.047565326 \\
\hline ESR $\S$ & 149 & 25 & $1.1(0.95-1.27)$ & 0.184748898 & 1 \\
\hline Triglycerides $\S$ & 163 & 30 & $1.05(0.99-1.11)$ & 0.11199928 & 1 \\
\hline Cholesterol $\S$ & 163 & 30 & 0.97 (0.89-1.07) & 0.59449302 & 1 \\
\hline LDH $\S$ & 155 & 31 & $0.99(0.96-1.02)$ & 0.623247956 & 1 \\
\hline CK $\S$ & 162 & 37 & $1(0.99-1.02)$ & 0.856646053 & 1 \\
\hline Troponin $\S$ & 101 & 24 & $1(0.99-1.01)$ & 0.750801089 & 1 \\
\hline D-dimer $\S$ & 119 & 25 & $1(1-1)$ & 0.029007362 & 1 \\
\hline Procalcitonin $\S$ & 118 & 24 & $1.75(0.89-3.47)$ & $1.06 \times 10^{-1}$ & 1 \\
\hline CRP $\S$ & 207 & 43 & $2.26(1.6-3.2)$ & $4.28496 \times 10^{-6}$ & 0.000205678 \\
\hline
\end{tabular}

† Number of subjects with information available; $\ddagger$ number of events (persons with delirium); ${ }^{*}$ Bonferroni adjustment; ^ HR per 1 unit increment; § HR per 10 units increment (units for each variable are listed in Table 2).

These predictors were then entered in a stepwise multivariable model which retained four independent variables. Presence of dementia was the strongest predictor of delirium, followed by age at admission, CRP, and GCS (Table 4). 
Table 4. Variables associated with delirium occurrence in the multivariable model.

\begin{tabular}{|c|c|c|}
\hline \multicolumn{3}{|c|}{ Occurrence of Delirium } \\
\hline Variable & $\begin{array}{c}\text { Hazard Ratio (95\% Confidence } \\
\text { Interval) }\end{array}$ & $p$-Value \\
\hline Age at admission ^ & $1.07(1.03-1.11)$ & 0.0007 \\
\hline Glasgow coma scale & $0.88(0.8-0.98)$ & 0.0166 \\
\hline Baseline CRP § & $1.06(1.02-1.1)$ & 0.0015 \\
\hline Dementia & $3.2(1.38-7.38)$ & 0.0065 \\
\hline
\end{tabular}

${ }^{\wedge}$ HR per 1 unit increment; § HR per 10 units increment.

Twenty-nine patients with delirium died during hospitalization and 10 were admit- ted to the ICU, compared with 26 and 30 patients without delirium, respectively. The presence of delirium increased the probability of death (Table 5) and the length of hospital stay for patients who survived, whereas the probability of admission to ICU and the length of stay in ICU were not affected (Table 5). To investigate whether delirium was independently associated with a poor outcome, we selected a composite outcome (death/admission to the ICU) and evaluated the probability to attain such an outcome in patients with and without delirium (Figure 1), and the predictive value of clinical and laboratory variables in a univariable model (Table 6). After adjustment for multiple comparisons, age, GCS, Charlson Index without dementia and a number of laboratory variables (hemoglobin, white blood cell count, neutrophils, eGFR, LDH, D-dimer, CRP, NLR) were found to be associated with the composite outcome.

Table 5. Association between delirium and outcomes.

\begin{tabular}{|c|c|c|c|}
\hline & $\begin{array}{c}\text { Patients with } \\
\text { Delirium }(N=46)\end{array}$ & $\begin{array}{l}\text { Patients without } \\
\text { Delirium }(N=168)\end{array}$ & $p$-Value \\
\hline $\begin{array}{c}\text { Length of hospitalization (only } \\
\text { survived patients days) (mean, SD) }\end{array}$ & 33.44 (12.63) & $24.64(13.28)$ & Poisson model, $p<0.0001$ \\
\hline Admission to ICU (N, \%) & $10 / 4621.7 \%$ & $30 / 16817.9 \%$ & $\begin{array}{c}\text { Cox model Hazard } \\
\text { Ratio }=1.26, p=0.53\end{array}$ \\
\hline $\begin{array}{l}\text { Time to ICU admission (only patients } \\
\text { admitted in ICU) days (mean, SD) }\end{array}$ & $10.60(15.04)$ & $6.90(7.40)$ & $\begin{array}{c}\text { Poisson model } \\
p<0.0005\end{array}$ \\
\hline $\begin{array}{l}\text { Length of stay in ICU (only patients } \\
\text { admitted in ICU) days (mean, SD) }\end{array}$ & $24.00(4.24)$ & $24.00(8.73)$ & $\begin{array}{l}\text { Poisson model, } \\
\quad p=0.893\end{array}$ \\
\hline In-hospital death $(\mathrm{N}, \%)$ & $29 / 4663.0 \%$ & $26 / 168$ 15.5\% & $\begin{array}{c}\text { Cox model Hazard } \\
\text { Ratio }=8.27, p<0.0001\end{array}$ \\
\hline
\end{tabular}

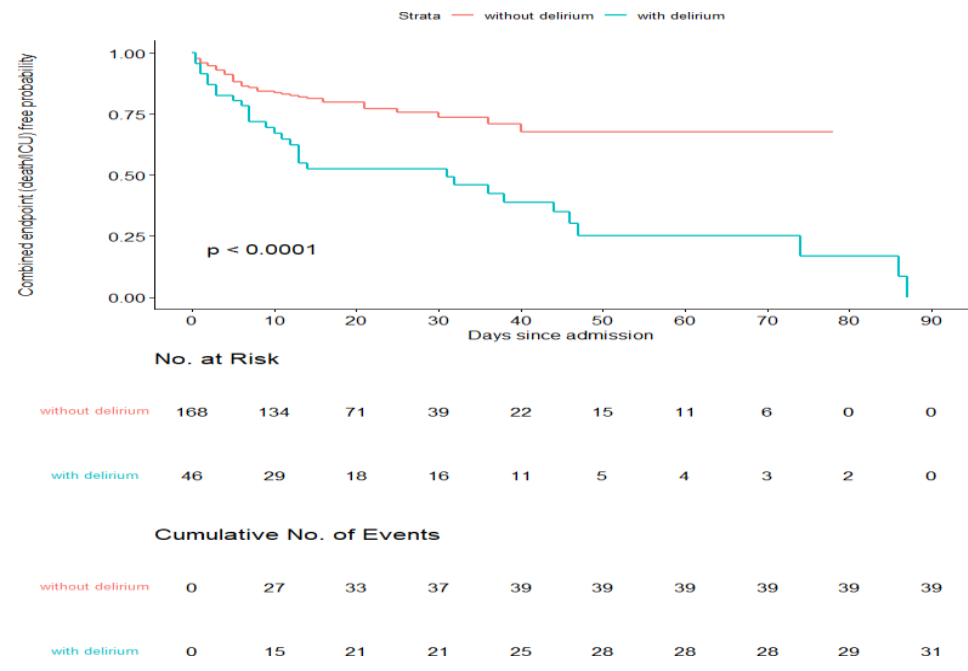

Figure 1. Kaplan-Meier survival curves according to presence/absence of delirium. 
Table 6. Association between baseline clinical and laboratory variables and the combined endpoint (death or admission to ICU).

\begin{tabular}{|c|c|c|c|c|c|}
\hline \multicolumn{6}{|c|}{ Combined Endpoint (Death/Admission to ICU) } \\
\hline Variable & $\mathbf{N}+$ & $\begin{array}{c}\mathbf{N} \\
\text { Events } \ddagger\end{array}$ & $\begin{array}{l}\text { Hazard Ratio (95\% } \\
\text { Confidence Interval) }\end{array}$ & $p$-Value & $p$-Value Adjusted * \\
\hline Delirium (time dependent) & 214 & 70 & $5.12(2.99-8.78)$ & 0.000000003 & 0.0000001 \\
\hline Age at admission & 214 & 70 & $1.05(1.03-1.08)$ & 0.000000237 & 0.0000116 \\
\hline Male gender & 214 & 70 & $1.05(0.65-1.69)$ & 0.837643647 & 1.0000000 \\
\hline Current/former smokers & 162 & 50 & $1.12(0.59-2.13)$ & 0.722477102 & 1.0000000 \\
\hline $\mathrm{BMI}^{\wedge}$ & 192 & 58 & $1.01(1-1.01)$ & 0.121131099 & 1.0000000 \\
\hline Glasgow Coma Scale ^ & 205 & 65 & $0.86(0.8-0.93)$ & 0.000145628 & 0.0071358 \\
\hline Fever & 214 & 70 & $0.69(0.42-1.13)$ & 0.142083876 & 1.0000000 \\
\hline Chronic Pulmonary & & & & & \\
\hline $\begin{array}{l}\text { Obstructive } \\
\text { Disease }\end{array}$ & 214 & 70 & $2.15(1.19-3.88)$ & 0.011525366 & 0.5647430 \\
\hline Diabetes & 214 & 70 & $1.39(0.8-2.41)$ & 0.246348210 & 1.0000000 \\
\hline Atrial fibrillation & 214 & 70 & $1.65(0.91-2.97)$ & 0.096134852 & 1.0000000 \\
\hline Arterial hypertension & 214 & 70 & $1.7(1.05-2.76)$ & 0.030715219 & 1.0000000 \\
\hline Hypercholesterolemia & 214 & 70 & $0.47(0.15-1.5)$ & 0.200883917 & 1.0000000 \\
\hline Tumor & 214 & 70 & $1.74(1.02-2.95)$ & 0.041879511 & 1.0000000 \\
\hline Thyreopathy & 214 & 70 & $1.19(0.51-2.76)$ & 0.684096031 & 1.0000000 \\
\hline Hypertriglyceridemia & 214 & 70 & $0.79(0.19-3.21)$ & 0.738138639 & 1.0000000 \\
\hline Other comorbidities & 214 & 70 & $2.65(1.45-4.84)$ & 0.001604357 & 0.0786135 \\
\hline $\begin{array}{c}\text { Charlson Index (without } \\
\text { dementia) }\end{array}$ & 214 & 70 & $1.28(1.16-1.41)$ & 0.000000394 & 0.0000193 \\
\hline Use of psychotropic drugs & 214 & 70 & $1.78(0.97-3.25)$ & 0.062649518 & 1.0000000 \\
\hline Dementia & 213 & 69 & $2.36(1.39-4.03)$ & 0.001521415 & 0.0745493 \\
\hline Vit. D therapy & 214 & 70 & $1.21(0.38-3.85)$ & 0.750983797 & 1.0000000 \\
\hline $\begin{array}{l}\text { Neutrophil/lymphocyte } \\
\text { ratio } \S\end{array}$ & 210 & 67 & $1.57(1.37-1.81)$ & 0.000000000 & 0.0000000 \\
\hline Hematocrit § & 210 & 67 & $0.62(0.44-0.89)$ & 0.009681733 & 0.4744049 \\
\hline Hemoglobin § & 210 & 67 & $0.15(0.05-0.45)$ & 0.000721321 & 0.0353447 \\
\hline MCV $\S$ & 204 & 62 & $1.24(0.89-1.73)$ & 0.202686714 & 1.0000000 \\
\hline $\mathrm{RBC} \S$ & 210 & 67 & $0.07(0-1.61)$ & 0.096831372 & 1.0000000 \\
\hline WBC $\S$ & 210 & 67 & $1.55(1.24-1.94)$ & 0.000139079 & 0.0068149 \\
\hline Neutrophils $\S$ & 210 & 67 & $1.22(1.09-1.37)$ & 0.000454567 & 0.0222738 \\
\hline Lymphocytes § & 210 & 67 & $0.99(0.54-1.8)$ & 0.972133027 & 1.0000000 \\
\hline Platelets § & 210 & 67 & $1(0.98-1.03)$ & 0.880538484 & 1.0000000 \\
\hline INR § & 194 & 60 & $10.95(0.63-189.64)$ & 0.099877021 & 1.0000000 \\
\hline PTT § & 194 & 60 & $1.52(0.96-2.41)$ & 0.075694508 & 1.0000000 \\
\hline ALT $\S$ & 205 & 65 & $1.01(0.97-1.04)$ & 0.702865821 & 1.0000000 \\
\hline Total bilirubin $\S$ & 202 & 62 & $3.29(0.19-55.53)$ & 0.408924418 & 1.0000000 \\
\hline Alkaline phosphatase $\S$ & 163 & 52 & $1.05(1.01-1.09)$ & 0.014802566 & 0.7253258 \\
\hline y-GT $\S$ & 164 & 53 & $1.01(0.99-1.03)$ & 0.329223790 & 1.0000000 \\
\hline AST § & 205 & 65 & $1.02(1-1.05)$ & 0.071553567 & 1.0000000 \\
\hline Total proteins $\S$ & 203 & 63 & $0.02(0-0.3)$ & 0.005466919 & 0.2678790 \\
\hline Creatinine $\S$ & 206 & 65 & $1.36(0.81-2.3)$ & 0.250416795 & 1.0000000 \\
\hline e-GFR § & 202 & 65 & $0.89(0.83-0.95)$ & 0.000202712 & 0.0099329 \\
\hline Glycemia § & 204 & 64 & $1.05(1.02-1.08)$ & 0.002704169 & 0.1325043 \\
\hline ESR $\S$ & 149 & 42 & $1.08(0.96-1.2)$ & 0.184670429 & 1.0000000 \\
\hline Triglycerides $\S$ & 163 & 49 & $1(0.95-1.05)$ & 0.994884031 & 1.0000000 \\
\hline Cholesterol § & 163 & 49 & $0.93(0.86-1.01)$ & 0.087372174 & 1.0000000 \\
\hline LDH § & 155 & 45 & $1.03(1.02-1.05)$ & 0.000171386 & 0.0083979 \\
\hline $\mathrm{CK} \S$ & 162 & 55 & $1(0.99-1.02)$ & 0.578550518 & 1.0000000 \\
\hline Troponin § & 101 & 29 & $1(1-1)$ & 0.033795411 & 1.0000000 \\
\hline D-dimer § & 119 & 35 & $1(1-1)$ & 0.000014371 & 0.0007042 \\
\hline Procalcitonin $\S$ & 118 & 38 & $1.02(0.49-2.16)$ & 0.949739846 & 1.0000000 \\
\hline CRP \& & 207 & 65 & $2.37(1.76-3.19)$ & 0.000000013 & 0.0000006 \\
\hline
\end{tabular}

† Number of subjects with information available; $\ddagger$ number of events (persons with death/admission to ICU); * Bonferroni adjustment; ${ }^{\wedge}$ HR per 1 unit increment; $§$ HR per 10 units increment (units for each variable are listed in Table 2).

These predictors entered the stepwise multivariable model, which retained delirium as the strongest independent predictor (Table 7) of death/admission to ICU, together with only three other variables: Charlson Index without dementia, CRP and NLR. After dividing delirium by clinical type, the probability of death/admission to ICU was higher for patients 
with the hypoactive subtype than for those with the hyperactive subtype; patients with the mixed subtype had an intermediate risk (Table 7).

Table 7. Predictors of poor outcome (death or admission to ICU) in the multivariable model.

\begin{tabular}{|c|c|c|c|c|}
\hline \multirow[b]{2}{*}{ Variable } & \multicolumn{2}{|c|}{ Model A } & \multicolumn{2}{|c|}{ Model B } \\
\hline & $\begin{array}{l}\text { Hazard Ratio (95\% } \\
\text { Confidence Interval) }\end{array}$ & $p$-Value & $\begin{array}{l}\text { Hazard Ratio (95\% } \\
\text { Confidence Interval) }\end{array}$ & $p$-Value \\
\hline $\begin{array}{c}\text { Neutrophils-to-lymphocytes } \\
\text { ratio } \S\end{array}$ & $1.03(1.01-1.05)$ & 0.001443974 & $1.03(1.01-1.05)$ & 0.001066611 \\
\hline Baseline CRP $\S$ & $1.07(1.03-1.1)$ & 0.000282734 & $1.06(1.02-1.1)$ & 0.001314788 \\
\hline $\begin{array}{l}\text { Charlson Index } \\
\text { (without dementia) }\end{array}$ & $1.21(1.07-1.36)$ & 0.001655282 & $1.22(1.08-1.38)$ & 0.000977608 \\
\hline Delirium & $3.81(2.15-6.73)$ & $4.25597 \times 10^{-6}$ & & \\
\hline $\begin{array}{l}\text { Hyperactive delirium } \\
\qquad(=15)\end{array}$ & & & $2.09(0.64-6.83)$ & 0.2199668 \\
\hline $\begin{array}{l}\text { Hypoactive delirium } \\
\qquad(\mathrm{N}=21)\end{array}$ & & & $5.95(2.85-12.42)$ & $2.05376 \times 10^{-6}$ \\
\hline Mixed delirium $(\mathrm{N}=11)$ & & & $3.02(1.08-8.46)$ & 0.03588895 \\
\hline
\end{tabular}

Reference category is no delirium. Model A: any type of delirium; Model B: separately evaluates hypo-, hyperactive or mixed; § HR for 10 units increment (units for each variable are listed in Table 2).

\section{Discussion}

In this observational study on consecutive patients admitted to the emergency department of a general hospital, 22\% of 214 patients with COVID-19 infection developed delirium during hospital stay or immediately before admission. Factors independently associated with the risk of delirium included older age, GCS, presence of dementia, and CRP. In turn, delirium was the strongest independent predictor of admission to ICU or intra-hospital death.

Prevalence: The prevalence of delirium in COVID-19 patients depends on the study population, diagnostic modality, and setting. It is higher in the ICU setting, ranging from 65 to $80 \%$, than in the general wards [14]. Comparison is made difficult by the variety of definitions of delirium (confusion, altered mental status, acute onset of psychotic symptoms, disorientation, decreased level of consciousness, cognitive dysfunction, and encephalopathy) [15] and of modalities of ascertainment. We defined delirium based on a two-step approach and according to DSM-5, whereas most studies relied on the review of medical records or did not include psychiatric evaluation. We found a prevalence in the range of that observed in COVID-19 patients admitted to general medicine wards (6 to 67\%) [14] and similar to the percentage of $23 \%$ reported in a meta-analysis of 33 studies on pre-COVID- 19 inpatients [2]. Other series in the same setting reported prevalence ratios of delirium almost three times higher in patients with COVID-19 than in those without [15,16]; however, in these series, the prevalence in non-COVID-19 control patients (range $=5.0-7.7 \%$ ) seems largely underestimated. Unfortunately, we do not have a comparison series of non-COVID patients, and most other studies are also lacking setting- and age-matched controls.

Outcomes: Delirium is independently associated with multiple poor outcomes in nonCOVID-19 patients [2], especially in its hypoactive subtype. Delirium was the strongest predictor of a composite outcome (death/admission to the ICU) in our series. In the final multivariable model, adjusting for several other covariates, patients with delirium had an almost fourfold probability of reaching the composite outcome, regardless of other factors. The risk was substantially higher for the hypoactive subtype. Delirium was not included in the majority of studies exploring predictive factors for mortality in COVID-19 patients [17] A meta-analysis of nine studies [18] showed that the mortality rate of COVID-19 patients with delirium was more than twice the mortality rate of those without delirium. In addition to delirium, only three other factors were identified as predictors of death in COVID-19 patients by our multivariable model: comorbidity measured with the Charlson Index, CRP, and NLR. In COVID-19 patients, comorbidity, age, and male sex were the major predictors 
of death in two large studies [19-21], and three meta-analyses [17,22,23] found CRP and NLR as predictors of severity and intra-hospital death as well.

Factors associated with delirium: If delirium has such a strong predictive value for poor outcome in COVID-19 patients admitted to general medicine wards, the search for factors predisposing patients to its occurrence (our second question) is of crucial clinical importance. In this regard, there is only sparse evidence in the literature. In a multicenter study including 817 older patients, significant risk factors included age $>75$ years, prior psychoactive medication use, comorbidities, and cognitive impairment or dementia [24]. These predisposing factors for delirium in COVID-19 patients overlap with those observed in the pre-COVID era [2]. We examined several clinical and laboratory features at hospital admission as possibly associated with delirium. After adjustment for multiple comparisons, we found that only age, GCS, dementia, Charlson Index without dementia, pre-admission use of psychotropic drugs, CRP and NLR were associated with delirium in the univariable analysis. In particular, NLR and CRP were associated with an increased risk of delirium per ten units of measurement by $45 \%$ and $126 \%$, respectively. In the multivariable analysis, only four variables were retained in the model as independently associated: dementia showed the highest predictive value, followed by age at admission, CRP, and GCS. NLR was excluded because of collinearity with CRP, most likely because both are markers of the same process, systemic inflammation. Additionally, comorbidity was removed from the final model because of collinearity with age and dementia. This result is consistent with previous evidence in the non-COVID-19 literature, which indicates that CRP independently predicts delirium [25-27], and with recent findings in COVID-19 patients suggesting an association between CRP levels and delirium occurrence [28]. The possible pathophysiologic mechanisms (direct invasion of the brain, brain hypoxia due to systemic hypoxemia or coagulopathy, cytokine storm and neuroinvasion) [29-31] that contribute to the occurrence of delirium among COVID-19 patients, and their mutual interaction, are still unclear. Both CRP and NLR are markers of systemic inflammation, thus supporting the hypothesis that inflammation has a role in the pathophysiology of delirium in COVID-19 patients. Systemic inflammatory mediators cross the blood-brain barrier, activate brain microglia, and initiate neuroinflammation, probably through some blood-brain barrier disruption [29,32]. In this scenario, individuals predisposed to a heightened inflammatory response when exposed to an acute stressor are at increased risk of delirium [26].

Strengths and Limitations: Strengths of this study include its external validity because of consecutive patient enrollment and its general hospital setting, a high level of delirium diagnostic certainty because of a two-step evaluation, and the inclusion of different putative risk factors associated with delirium. Three limitations are important to mention: first, some data, including education, living situation and socioeconomic status, were missing from the record. These factors may limit the generalizability of the findings. Second, we analyzed predictive factors for delirium in patients admitted to a general ward, while they may be different in another setting, such as the ICU [6]. Third, our outcome measure combined death and admission to the ICU; more frail patients, such as those with delirium, may be less likely to be considered for admission to the ICU, and this could have underestimated their risk to achieve the outcome.

\section{Conclusions}

In conclusion, we found a few clinical features and inflammatory markers associated with delirium in COVID-19 patients. Although they are far too non-specific to be considered as markers for the occurrence of delirium, they can be easily ascertained at admission and used as a proxy for stratifying the risk of developing delirium in COVID-19 patients. In daily clinical practice, identifying COVID-19 patients at risk of delirium can be very challenging. We believe that the clinical and inflammatory markers identified in this study may help clinicians to select patients who would benefit from both non-pharmacological and pharmacological interventions in order to prevent delirium, and in turn to reduce the risk of admission to ICU or death. 
Author Contributions: M.A.L., A.D.G., A.M., F.A., A.G., M.C. (Massimiliano Copetti) and S.D.C. planned the study, analyzed and interpreted the data, and wrote the first draft of the paper. A.D.G., C.D.G., P.E.A., L.F., V.I., M.Z., G.M. and A.A. collected the data. P.R., C.M. and M.C. (Massimo Carella) worked on database implementation. M.C. (Massimiliano Copetti) and A.F. performed the statistical analysis. The author listed in the group collected data. All authors revised the drafts, and approved the final version of the paper. All authors had full access to all data in the study, including statistical reports and tables, and can take responsibility for the integrity of data and accuracy of data analysis. All authors have read and agreed to the published version of the manuscript.

Funding: This research received no external funding.

Institutional Review Board Statement: Ethical Committee of the Fondazione Casa Sollievo della Sofferenza, Protocol COVID-19-SGR, num. 46/2020.

Informed Consent Statement: Informed consent was obtained from all subjects involved in the study.

Data Availability Statement: The data presented in this study are available on request from the corresponding author. The data are not publicly available due to ethical and privacy restrictions.

Acknowledgments: The authors thank Chiara Di Giorgio (Fondazione IRCCS Casa Sollievo della Sofferenza) for proofreading and editing of the text.

Conflicts of Interest: The authors report no conflict of interest, and that the funders of the study had no role in the study design, data collection, data analysis, data interpretation, or writing of the report.

\section{Appendix A}

Other members of the CSS-COVID-19 Group are: Annalucia Biancofiore, Cristina Borelli, Cristiano Carbonelli, Giulia Castorani, Marco Castori, Antonella Centonze, Gianluca L. Ciliberti, Michele Corritore, Leonardo D'Aloiso, Maria M. D'Errico, Angela de Matthaeis, Alfredo Del Gaudio, Lazzaro Di Mauro, Vincenzo Giambra, Elvira Grandone, Michele Inglese, Maria Labonia, Antonella La Marca, Tiziana Latiano, Renato Lombardi, Evaristo Maiello, Alessandra Mangia, Valentina Massa, Simonetta Massafra, Grazia Vittoria Orciulo, Nicola Palladino, Rita Perna, Pamela Piscitelli, Matteo Piemontese, Michele A. Prencipe, Maria Grazia Rodriquenz, Raffaele Russo, Daniele Sancarlo, Anna Simeone, Vincenzo Trischitta, Pasquale Vaira, Doriana Vergara, Angelo Vescovi.

\section{References}

1. American Psychiatric Association. Diagnostic and Statistical Manual of Mental Disorders, 5th ed.; American Psychiatric Association: Arlington, VA, USA, 2013.

2. Wilson, J.E.; Mart, M.F.; Cunningham, C.; Shehabi, Y.; Girard, T.D.; MacLullich, A.M.J.; Slooter, A.J.C.; Ely, E.W. Delirium. Nat. Rev. Dis. Primers 2020, 6, 90; Erratum in Nat. Rev. Dis. Primers 2020, 6, 94. [CrossRef] [PubMed]

3. D'Ardes, D.; Carrarini, C.; Russo, M.; Dono, F.; Speranza, R.; Digiovanni, A.; Martinotti, G.; Di Iorio, A.; Onofrj, M.; Cipollone, F.; et al. Low molecular weight heparin in COVID-19 patients prevents delirium and shortens hospitalization. Neurol. Sci. 2021, 42, 1527-1530. [CrossRef] [PubMed]

4. Rogers, J.P.; Chesney, E.; Oliver, D.; Pollak, T.A.; McGuire, P.; Fusar-Poli, P.; Zandi, M.S.; Lewis, G.; David, A.S. Psychiatric and neuropsychiatric presentations associated with severe coronavirus infections: A systematic review and meta-analysis with comparison to the COVID-19 pandemic. Lancet Psychiatry 2020, 7, 611-627. [CrossRef]

5. Shao, S.C.; Lai, C.C.; Chen, Y.H.; Chen, Y.C.; Hung, M.J.; Liao, S.C. Prevalence, incidence and mortality of delirium in patients with COVID-19: A systematic review and meta-analysis. Age Ageing 2021, 50, 1445-1453. [CrossRef]

6. Pun, B.T.; Badenes, R.; Heras La Calle, G.; Orun, O.M.; Chen, W.; Raman, R.; Simpson, B.K.; Wilson-Linville, S.; Hinojal Olmedillo, B.; de la Cueva, A.V.; et al. COVID-19 Intensive Care International Study Group. Prevalence and risk factors for delirium in critically ill patients with COVID-19 (COVID-D): A multicentre cohort study. Lancet Respir. Med. 2021, 9, 239-250. [CrossRef]

7. Lou, B.; Guo, J.; Liu, Y.; Xiong, C.; Shi, J.; Zhou, C. Prevalence, classification, risk factors and outcome impact of delirium in patients with COVID-19: A meta-analysis protocol for systematic review. BMJ Open 2021, 11, e048323. [CrossRef]

8. O'Hanlon, S.; Inouye, S.K. Delirium: A missing piece in the COVID-19 pandemic puzzle. Age Ageing 2020, 49, 497-498. [CrossRef]

9. Vabret, N.; Britton, G.J.; Gruber, C.; Hegde, S.; Kim, J.; Kuksin, M.; Levantovsky, R.; Malle, L.; Moreira, A.; Park, M.D.; et al. Sinai Immunology Review Project. Immunology of COVID-19: Current State of the Science. Immunity 2020, 52, 910-941. [CrossRef] 
10. Mirijello, A.; Zarrelli, M.; Miscio, G.; de Matthaeis, A.; Piscitelli, P.; Carbonelli, C.M.; Di Giorgio, A.; Inglese, M.; Ciliberti, G.L.; Marciano, C.; et al. Diagnosis of COVID-19 in Patients with Negative Nasopharyngeal Swabs: Reliability of Radiological and Clinical Diagnosis and Accuracy Versus Serology. Diagnostics 2021, 11, 386. [CrossRef]

11. Charlson, M.; Szatrowski, T.P.; Peterson, J.; Gold, J. Validation of a combined comorbidity index. J. Clin. Epidemiol. 1994, 47, 1245-1251. [CrossRef]

12. Bellelli, G.; Morandi, A.; Davis, D.H.; Mazzola, P.; Turco, R.; Gentile, S.; Ryan, T.; Cash, H.; Guerini, F.; Torpilliesi, T.; et al. Validation of the 4AT, a new instrument for rapid delirium screening: A study in 234 hospitalised older people. Age Ageing 2014, 43, 496-502, Erratum in Age Ageing 2015, 44, 175. [CrossRef] [PubMed]

13. Lipowski, Z.J. Delirium: Acute Confusional States; Oxford University Press: New York, NY, USA, 1990.

14. Hawkins, M.; Sockalingam, S.; Bonato, S.; Rajaratnam, T.; Ravindran, M.; Gosse, P.; Sheehan, K.A. A rapid review of the pathoetiology, presentation, and management of delirium in adults with COVID-19. J. Psychosom. Res. 2021, 141, 110350. [CrossRef] [PubMed]

15. Slooter, A.J.C.; Otte, W.M.; Devlin, J.W.; Arora, R.C.; Bleck, T.P.; Claassen, J.; Duprey, M.S.; Ely, E.W.; Kaplan, P.W.; Latronico, N.; et al. Updated nomenclature of delirium and acute encephalopathy: Statement of ten Societies. Intensive Care Med. 2020, 46, 1020-1022. [CrossRef] [PubMed]

16. Pilotto, A.; Benussi, A.; Libri, I.; Masciocchi, S.; Poli, L.; Premi, E.; Alberici, A.; Baldelli, E.; Bonacina, S.; Brambilla, L.; et al. COVID-19 impact on consecutive neurological patients admitted to the emergency department. J. Neurol. Neurosurg. Psychiatry 2021, 92, 218-220. [CrossRef] [PubMed]

17. Benussi, A.; Pilotto, A.; Premi, E.; Libri, I.; Giunta, M.; Agosti, C.; Alberici, A.; Baldelli, E.; Benini, M.; Bonacina, S.; et al. Clinical characteristics and outcomes of inpatients with neurologic disease and COVID-19 in Brescia, Lombardy, Italy. Neurology 2020, 95, e910-e920. [CrossRef] [PubMed]

18. Wu, Y.; Li, H.; Zhang, Z.; Liang, W.; Zhang, T.; Tong, Z.; Guo, X.; Qi, X. Risk factors for mortality of coronavirus disease 2019 (COVID-19) patients during the early outbreak of COVID-19: A systematic review and meta-analysis. Ann. Palliat. Med. 2021, 10, 5069-5083. [CrossRef] [PubMed]

19. Pranata, R.; Huang, I.; Lim, M.A.; Yonas, E.; Vania, R.; Kuswardhani, R.A.T. Delirium and Mortality in Coronavirus Disease 2019 (COVID-19)_A Systematic Review and Meta-analysis. Arch. Gerontol. Geriatr. 2021, 95, 104388. [CrossRef]

20. Williamson, E.J.; Walker, A.J.; Bhaskaran, K.; Bacon, S.; Bates, C.; Morton, C.E.; Curtis, H.J.; Mehrkar, A.; Evans, D.; Inglesby, P.; et al. Factors associated with COVID-19-related death using OpenSAFELY. Nature 2020, 584, 430-436. [CrossRef]

21. Docherty, A.B.; Harrison, E.M.; Green, C.A.; Hardwick, H.E.; Pius, R.; Norman, L.; Holden, K.A.; Read, J.M.; Dondelinger, F.; Carson, G.; et al. ISARIC4C investigators. Features of 20133 UK patients in hospital with covid-19 using the ISARIC WHO Clinical Characterisation Protocol: Prospective observational cohort study. BMJ 2020, 369, m1985. [CrossRef]

22. Ali, N. Elevated level of C-reactive protein may be an early marker to predict risk for severity of COVID-19. J. Med. Virol. 2020, 92, 2409-2411. [CrossRef]

23. Simadibrata, D.M.; Calvin, J.; Wijaya, A.D.; Ibrahim, N.A.A. Neutrophil-to-lymphocyte ratio on admission to predict the severity and mortality of COVID-19 patients: A meta-analysis. Am. J. Emerg. Med. 2021, 42, 60-69. [CrossRef] [PubMed]

24. Kennedy, M.; Helfand, B.K.I.; Gou, R.Y.; Gartaganis, S.L.; Webb, M.; Moccia, J.M.; Bruursema, S.N.; Dokic, B.; McCulloch, B.; Ring, H.; et al. Delirium in Older Patients With COVID-19 Presenting to the Emergency Department. JAMA Netw. Open 2020, 3, e2029540. [CrossRef] [PubMed]

25. Macdonald, A.; Adamis, D.; Treolar, A.; Martin, F. C-reactive protein levels predict incidence of and recovery from delirium. Age Ageing 2007, 36, 222-225. [CrossRef] [PubMed]

26. Vasunilashorn, S.M.; Dillon, S.T.; Inouye, S.K.; Ngo, L.H.; Fong, T.G.; Jones, R.N.; Travison, T.G.; Schmitt, E.M.; Alsop, D.C.; Freedman, S.D.; et al. High C-Reactive Protein Predicts Delirium Incidence, Duration, and Feature Severity After Major Non-cardiac Surgery. J. Am. Geriatr. Soc. 2017, 65, e109-e116. [CrossRef]

27. Dillon, S.T.; Vasunilashorn, S.M.; Ngo, L.; Otu, H.H.; Inouye, S.K.; Jones, R.N.; Alsop, D.C.; Kuchel, G.A.; Metzger, E.D.; Arnold, S.E.; et al. Higher C-Reactive Protein Levels Predict Postoperative Delirium in Older Patients Undergoing Major Elective Sur- gery: A Longitudinal Nested Case-Control Study. Biol. Psychiatry 2017, 81, 145-153. [CrossRef]

28. Saini, A.; Oh, T.H.; Ghanem, D.A.; Castro, M.; Butler, M.; Sin Fai Lam, C.C.; Posporelis, S.; Lewis, G.; David, A.S.; Rogers, J.P. Inflammatory and blood gas markers of COVID-19 delirium compared to non-COVID-19 delirium: A cross-sectional study. Aging Ment. Health 2021, 1-8. [CrossRef]

29. Kealy, J.; Murray, C.; Griffin, E.W.; Lopez-Rodriguez, A.B.; Healy, D.; Tortorelli, L.S.; Lowry, J.P.; Watne, L.O.; Cunningham, C. Acute Inflammation Alters Brain Energy Metabolism in Mice and Humans: Role in Suppressed Spontaneous Activity, Impaired Cognition, and Delirium. J. Neurosci. 2020, 40, 5681-5696. [CrossRef]

30. Tremblay, M.E.; Madore, C.; Bordeleau, M.; Tian, L.; Verkhratsky, A. Neuropathobiology of COVID-19: The Role for Glia. Front. Cell. Neurosci. 2020, 14, 592214. [CrossRef]

31. Boldrini, M.; Canoll, P.D.; Klein, R.S. How COVID-19 Affects the Brain. JAMA Psychiatry 2021, 78, 682-683. [CrossRef]

32. Khan, B.A.; Perkins, A.J.; Prasad, N.K.; Shekhar, A.; Campbell, N.L.; Gao, S.; Wang, S.; Khan, S.H.; Marcantonio, E.R.; Twigg, H.L., 3rd; et al. Biomarkers of Delirium Duration and Delirium Severity in the ICU. Crit. Care Med. 2020, 48, 353-361. [CrossRef] 